\title{
Central sleep apnea - a case report
}

\author{
Andrzej Dybala ${ }^{1}$, Monika Dyczko ${ }^{1}$, Boguslaw Makaruk ${ }^{1}$, Pawel Kicinski ${ }^{1}$, \\ Elzbieta BartoszeK ${ }^{1}$, Wojciech Myslinski ${ }^{1}$, Mansur Rahnama ${ }^{2 *}$, Jerzy Mosiewicz ${ }^{1}$ \\ ${ }^{1}$ Department and Clinic of Internal Medicine, Medical University of Lublin, Poland \\ ${ }^{2}$ Chair and Department of Oral Surgery, Medical University of Lublin, Karmelicka 7, 20-081 Lublin, Poland
}

\section{ARTICLE INFO \\ Received 10 November 2013 \\ Accepted 03 February 2014}

\section{Keywords:}

entral sleep apnea,

heart failure,

BiPAP ST,

servo-ventilation.

\begin{abstract}
Central sleep apnea (CSA) is a disease characterized by repetitive episodes of the socalled central apneas during sleep. The disease has a very complex etiology. In clinical practice, the most important causes of CSA are disorders of the central nervous system, congestive heart failure or certain pathological changes of the respiratory muscles. We present a case of a 43-year-old male with severe CSA, who was successfully treated with BiPAP ST equipment.
\end{abstract}

\section{INTRODUCTION}

Central sleep apnea (CSA) is a disease of diverse etiology. The core of this disorder are episodes of so called central apnea that keep recurring during sleep. Polysomnography shows that during these episodes, the air flow through the airways ceases and so does, at the same time, respiratory effort in the rib cage and the abdomen, which results in reduction of oxygen saturation of haemoglobin. There is little reliable epidemiological data on the incidence of CSA in particular populations. This disease stays, as it were, in the background of the better-known disease, which is obstructive sleep apnea (OSA). It is more difficult for patients with central breathing disorders to receive effective medical treatment. In this study, we present a case of a patient with central sleep apnea and discuss the possible causes and therapy options for this kind of sleep-related breathing disorder.

\section{CASE REPORT}

A 43-year-old patient presented with a history of cardiac conditions and surgery operations, including myocardial infarction complicated with circulatory arrest, angioplasty of the right coronary artery and the LAD branch of the left coronary artery performed in 2007 , and implantation of a cardioverter-defibrillator in secondary prevention of sudden cardiac death. For many years, the patient had been treated with insulin for Type 2 diabetes. Admitted to the Clinic

\footnotetext{
Corresponding author

* e-mail:rahnama@plusnet.pl

tel.: +48 81 528-79-50

fax: 81 528-79-51
}

of Internal Medicine, Medical University of Lublin, with weakness, excessive daytime sleepiness (Epworth Sleepiness Scale score was 15), loud snoring and pauses in breathing observed by the patient's family. These symptoms persisted for 4-5 years and intensified a few months prior the hospitalization. In addition, the patient reported worsened metabolic control of diabetes. For about one year, the blood glucose level in self-control remained had remained around 150-350 $\mathrm{mg} / \mathrm{dl}$. Apart from central-type obesity (BMI-40 kg/m²), physical examination did not reveal any clinically significant abnormalities. ENT examination did not reveal any abnormalities, either. Laboratory tests revealed an elevated serum glucose level (172 mg/d) and slight glycosuria. Glycated haemoglobin (HbA1c) was $8.4 \%$. No clinically significant deviations in terms of CBC parameters, lipoprotein profile and concentration of electrolytes, urea, creatinine and TSH in blood serum were found. The serum level of NTproBNP was slightly elevated $(244 \mathrm{pg} / \mathrm{ml}$; normal is below $177 \mathrm{pg} / \mathrm{ml}$ ). In consideration of the symptoms reported by the patient and of the entirety of the clinical picture, the patient was subjected to polysomnography, during which 426 sleep apnea episodes were recorded. A straight majority of them - as many as 382 episodes of sleep apnea were of central nature, 35 episodes were mixed, and 9 - obstructive. Moreover, there were 160 occurrences of shallow breathing. The apnea hypopnea index was $73.3 / \mathrm{h}$. Most of the apnea episodes lasted 10-30 seconds; the longest episode of central apnea lasted 50 seconds. Sleep-related breathing disturbances triggered off clinically significant desaturation. Saturation below $90 \%$ had occurred for $52 \%$ of the sleep time. During the hospitalization, an attempt was made to determine the causes of central apnea. First of all, cardiac and neurological causes were taken into consideration. Echocardiography revealed impairment of the 
left ventricular global systolic function (ejection fraction was $42 \%$ ) as well as impaired left ventricular relaxation. Doppler ultrasound examination of carotid and vertebral arteries did not show any clinically significant lesions. Flow spectra were normal. During the hospitalization, a cranial CT scan was performed. It revealed intense cortical and subcortical atrophies, mainly within the frontal lobes. No other abnormalities that could be the causes of the sleep pathology were found. The patient was consulted by a neurologist, who found no traits of a recent lesion in the central and peripheral nervous system. The patient was offered a test continuous positive airway pressure therapy. Consideration was given to the use of an adaptive servo-ventilation (ASV) device, or, alternatively, a bilevel positive airway pressure device (BiPAP S/T) (rather the latter on account of the considerably lower purchase costs to be incurred by the patient). The patient decided to start his therapy using the BiPAP S/T device. Cessation of the breathing disturbances was achieved by modification of the device settings and confirmed in follow-up polysomnography. The patient remains under the care of the university outpatient clinic. At home, he uses a BiPAP S/T device. Central sleep apnea symptoms vanished. After about 2 months of the continuous positive airway pressure therapy, the patient's need for insulin decreased. The intensive insulin therapy scheme was modified to reduce especially the basal insulin doses. The metabolic control of diabetes gradually improved.

\section{DISCUSSION}

Breathing during sleep is subject to precise metabolic control. The control is regulated by chemoreceptors, which respond to changes in partial pressure of oxygen (carotid glomera) and of carbon dioxide and arterial blood $\mathrm{pH}$ (carotid glomera and respiratory centre receptors in the medulla) as well as by vagus nerves responding to the stimuli from the receptors in the walls of the rib cage, airways and lungs[1]. The neurotransmission disturbances in the reflex arch, to which contribute chemoreceptors, respiratory centre in the medulla and respiratory muscles, may cause shallow breathing and apnea [2,3]. Clinically, CSA can be caused by 3 essential groups of factors:

- central disturbances in the respiratory drive, secondary to central nervous system diseases, i.e. inflammations of the brain, damages to cervical segments of the spinal cord, cerebral infarctions and tumours,

- decreased respiratory muscle power, secondary to muscular dystrophies, myasthenia gravis, amyotrophic lateral sclerosis, phrenoplegia etc.

- cardiovascular system diseases - specifically congestive heart failure.

Sleep-related breathing disorders of central nature are a serious clinical problem for a broad group of patients with heart failure. According to various studies, central apnea and/or Cheyne-Stokes respiration episodes are present in $45-82 \%$ of patients with systolic heart failure [4]. They are a result of instability of the central respiratory drive. Reduced stroke blood volume and haemostasis in the ventricles extend the time needed by the blood to flow from the lungs, where gas exchange takes place, to the chemoreceptors sensitive to changes in oxygen and carbon dioxide pressure [5]. As a result, the chemoreceptors respond inadequately (to the actual composition of respiratory gases in the blood). When the partial pressure of carbon dioxide in the lungs drops, this state is interpreted, at the carotid glomus level, as a rise (due to the extended blood flow between the lungs and the chemoreceptors sensitive to $\mathrm{CO}_{2}$ concentration), which additionally intensifies the ventilation, bringing about significant hypocapnia with secondary inhibition of the respiratory centre and development of central apnea. Then, partial pressure of carbon dioxide in the lungs rises, and in the meantime, at the chemoreceptor level, it is still interpreted as its significant drop, which additionally extends the duration of the apnea episode. Depending on the span of the central hypoventilation phase, the polysomnogram shows central apnea episodes or Cheyne-Stokes (periodic) respiration episodes of different durations [3].

The result of multiple apnea and shallow breathing episodes that occur during sleep is hypoxia. In polysomnography, its exponent is the time, during which blood saturations falls below $90 \%$ (the so-called $t 90$ parameter). Oxygen deficiency has an adverse effect on the myocardium and reduces its contractility with time, thus deepening the heart failure. Recurring desaturation episodes activate sympathetic nerve fibres, which is reflected by high daily amounts of noradrenaline and its derivatives excreted in the urine by patients with central sleep apnea syndrome. This is one of the causes of serious and often treatment-resistant arterial hypertension. It is also a factor, which predisposes to cardiac rhythm disorders like atrial fibrillation, additional ventricular beats and ventricular tachycardia, which are a major cause of sleep-related deaths of patients with breathing disorders [1]. The hyperreactivity of the sympathetic system increases the heart muscle's oxygen demand, which in conditions of reduced oxygen supply in patients with breathing disturbances is a risk factor for ischemic heart disease. With the recurring cell shock episodes, the myocardium oxygen deficiency contributes to failure of the cells and reduction in the left ventricular ejection fraction. A drop in saturation is also a vasoconstrictive factor for the pulmonary arteries, causing pulmonary hypertension and, secondarily, right ventricular failure. Heart failure contributes to progression of the breathing disturbances in the mechanics described above.

Besides cardiac complications of the sleep apnea syndrome, hypoxia is also a causative factor in a number of endocrinological and metabolic disorders. As mentioned above, by intensifying the activity of the sympathetic system, hypoxia stimulates secretion of hormones that are antagonistic to insulin. The elevated levels of noradrenaline and its derivatives cause higher insulin demand and hyperinsulinemia as a result of hyperglycaemia. At the same time, as a stressful factor, hypoxia induces production of a number of pro-inflammatory cytokines, eg. interleukin 6 , and a tumour necrosis factor (TNF alfa), which are the factors that increase the resistance of soft and fatty tissues to insulin [10]. For this reason, both obstructive sleep apnea and central sleep apnea can, with time, cause Type 2 diabetes or worsen the metabolic control of previously diagnosed diabetes. 
In the clinical case discussed above, it was considerably difficult to determine the primary cause of the central sleep apnea episodes in the patient. The probable etiological factor determining the breathing disturbances in the patient might be post-infarction systolic heart failure. The other cortical and subcortical atrophies and functional changes that had occurred in the central nervous system structures playing a part in the breathing regulation most likely as a result of hypoxia related to sudden circulatory arrest, were also taken into consideration. Determination of the cause of central apnea is of great importance because the fundamental treatment of patients with CSA is always based on the therapy for the principal disease. For example, intensification of the heart failure therapy and improvement of the circulatory system function may result in relapse of the breathing disturbances [5]. In cases where it is impossible to explicitly determine or eliminate the cause, application of the positive airway pressure therapy during sleep is sometimes effective. The continuous positive airway pressure (CPAP) therapy is widely applied in patients with obstructive sleep apnea and constitutes the first choice method among patients with a serious form of OSA. Positive airway pressure provides patients with OSA with a "pneumatic skeleton" that prevents the upper airway lumen from sinking in during sleep, which leads to obstructive apnea episodes. Application of devices generating positive airway pressure is, by nature, very limited in cases of central breathing disorders. Some publications emphasize the therapeutic value of adaptive servoventilation. Automatically selecting pressure support, ASV devices effectively stop Cheyne-Stokes respiration, and in a situation of long central apnea episodes, they generate additional "rescue respiration" [6]. The use of BiPAP devices in the therapy still arouses controversy [7,8]. In Poland, they may be a certain alternative to servoventilation, chiefly because of the very high price of ASV devices (between ten and twenty thousand zlotys). One can certainly use the spontaneous/timed mode (S/T) that allows for generation of a minimum preset number of breaths. This is most likely done through the stimulation of the rib cage and airway mechanoreceptors by the positive pressure generated by the device [9].

More and more studies emphasize the fact that, besides the improvement in the patient's physical and mental state (cessation of daytime sleepiness), the positive airway pressure therapy brings notable cardiac and metabolic benefits. Effective treatment of central breathing disorders reduces the heart preload, improves myocardium contractility as well as reduces arterial blood pressure primarily through reduction of the sympathetic system activity [8]. Clinical data also show improvement in insulin sensitivity during CPAP therapy [10]. Such a phenomenon was also observed in the case analysed in present study.

\section{SUMMARY}

Central sleep apnea still constitutes an underrated clinical issue. Whereas, the range of this phenomenon is expanding, mainly due to ageing of and the heart failure prevalence in the population. In some patients with CSA, causal treatment may be applied. In cases of patients where causal treatment is not effective or infeasible, a positive airway pressure therapy, best with ASV devices, seems to be a valuable therapeutic option.

\section{REFERENCES}

1. Bradley T.D., Philipson E.A.: Central sleep apnea. Clin. Chest. Med., 13, 493-505, 1992.

2. Gozal T.D. et al.: Peripheral chemoreceptor function in children with the congenital hypoventilation syndrome. J. Appl. Physiol., 74, 379-387, 1993.

3. Bradley T.D.: Right and left ventricular functional impairment and sleep apnea. Clin. Chest. Med., 13, 459-479, 1992.

4. Garcia-Touchard A., Somers V., Olson L.J.: Central sleep apnea. Chest., 133(6), 1495-1504, 2008.

5. Malhotra A., Owens R.: What is central sleep apnea? Respir. Care., 55(9), 1168-1178, 2010.

6. Javaheri S. et al.: The performance of two automatic servo-ventilation devices in the treatment of central sleep apnea. SLEEP., 34(12): 1693$1698,2011$.

7. Randerath W. J.: Therapeutic options for the treatment of CheyneStokes respiration. Swiss. Med. Wkly., 138 (9-10); 135-139, 2009.

8. Arzt M. et al. Effects of dynamic bilevel positive airway pressure support on central sleep apnea in men with heart failure. Chest., 34(1):61-66, 2008.

9. Hormann C. et al.: Biphasic positive airway pressure (BIPAP)- a new mode of ventilatory support. Eur. J. Anaesthesiol., 11(1), 37-42, 1994.

10. Martins R.C., Andersen M.L., Tufik S.: The reciprocal interaction between sleep and type 2 diabetes mellitus: facts and perspectives. Braz. J. Med. Biol. Res., 41 (3): 180-187, 2008. 